PROTOCOL OPEN

\title{
Activating primary care COPD patients with multi-morbidity (APCOM) pilot project: study protocol
}

\author{
Sameera Ansari ${ }^{1}$, Hassan Hosseinzadeh ${ }^{1}$, Sarah Dennis ${ }^{2,3}$ and Nicholas Zwar ${ }^{1,4}$ \\ npj Primary Care Respiratory Medicine (2017)27:12 ; doi:10.1038/s41533-016-0003-9
}

\section{BACKGROUND}

Chronic obstructive pulmonary disease (COPD), third leading cause of mortality worldwide, ${ }^{1}$ is primarily caused by cigarette smoking in Australia. $^{2}$ COPD often occurs in the presence of multi-morbidity, which is the simultaneous occurrence of two or more chronic conditions; this is a growing concern in a health system focused on single-disease management. ${ }^{3}$ Around $80 \%$ of older Australians have multi-morbidity, average prevalence of chronic respiratory disease being $9.5 \%{ }^{4}$

A systematic review of COPD education programs suggests that equipping patients with self-management skills is as important as disease knowledge. ${ }^{5}$ Studies exploring positive effects of chronic disease-management interventions ${ }^{6}$ also underline the lack of studies aimed at empowering patients. Although a recent Cochrane Review ${ }^{7}$ of COPD self-management interventions shows increased health-related quality of life and decreased probability of respiratory-related hospitalisation, similar interventions have not been consistently effective when applied in recent trials. ${ }^{8-10}$ Though interventions aimed at modifying COPD-related health behaviour have great potential, extant literature points to need for self-management programs in primary care.

There is a dearth of interventions involving patients with multimorbidity, clinicians and other stakeholders, that can be generalisable to other populations. ${ }^{11}$ Patients with multimorbidity are often excluded from chronic disease-management trials; a Cochrane review ${ }^{12}$ of multi-morbidity interventions found only ten such studies. Our recent qualitative study, ${ }^{13}$ which explored the impact of COPD diagnosis in primary care patients with co-morbidities (co-existing chronic conditions), found suboptimal disease understanding and health-care utilisation. The above evidence demonstrates a need for development and testing interventions to increase self-management capacity in COPD patients with co-morbidities.

Even a brief education session is effective in improving disease knowledge and response to symptoms; ${ }^{14}$ as demonstrated by selfmanagement interventions ${ }^{10,15}$ on COPD which increased patient self-efficacy. Such programs do not only improve health status and prevent hospital remissions, ${ }^{16}$ but also lead to better patientprovider relationships, self-efficacy and sense of identity. ${ }^{17}$ With no prior study having seemed to focus on improving self-efficacy of COPD patients with co-morbidities in primary care, our study aims to empower these patients by trialling a tailored, practice nurse -delivered self-management program.

\section{AIMS}

This pre and post-test pilot study aims to trial the impact and feasibility of a tailored self-management program for primary care COPD patients with multi-morbidity. Our hypothesis is that at six months' follow-up, participants would have:

1. Better activation in terms of their COPD-related health behaviour.

2. Improved knowledge and self-management capacity of COPD.

3. Increased self-efficacy in terms of their overall health behaviours.

\section{METHODS}

This pilot study is funded by GlaxoSmithKline Australia and recognised by the National Health and Medical Research Council through a scholarship to S.A. The study has approval from the Human Research Ethics Committee of UNSW Australia (HREC14139).

Inclusion/exclusion criteria

General practices will be eligible if they maintain an electronic database of medical records and employ one or more practice nurse (PN).

Patients will be eligible if they are aged between 40 and 84 years, have a recorded spirometry diagnosis of COPD and at least one other co-morbidity. Exclusion criteria are poor understanding of English and/or cognitive impairment.

\section{Setting}

The study will be conducted in general practices across Sydney, Australia. Practices will be recruited from the UNSW Practice Based Research Network that links practices, which have expressed interest and/or have participated in previous research by S.D. and N.Z.

This is a pre and post-test study comprising a mixed method design, with outcome measures to be collected at baseline and six months' follow-up. The protocol is summarised in Fig. 1.

\section{Recruitment}

An information sheet about the study will be faxed to invite potential practices. S.A. will visit interested practices to answer questions and obtain written informed consent from general physician (GP)s and PNs. Practices who do not respond will be followed up after two weeks.

\footnotetext{
${ }^{1}$ School of Public Health and Community Medicine, UNSW Medicine, UNSW Australia, Sydney, NSW 2052, Australia; ${ }^{2}$ South Western Sydney Local Health District, Faculty of Health Sciences, The University of Sydney, Sydney, NSW 2141, Australia; ${ }^{3}$ Centre for Primary Health Care and Equity, UNSW Australia, Sydney, NSW 2052, Australia and ${ }^{4}$ School of Medicine, Faculty of Science, Medicine and Health, University of Wollongong Australia, Wollongong, NSW 2522, Australia

Correspondence: Sameera Ansari (sameera.ansari@unsw.edu.au)
}

Received: 14 April 2016 Revised: 11 September 2016 Accepted: 7 November 2016

Published online: 16 February 2017 


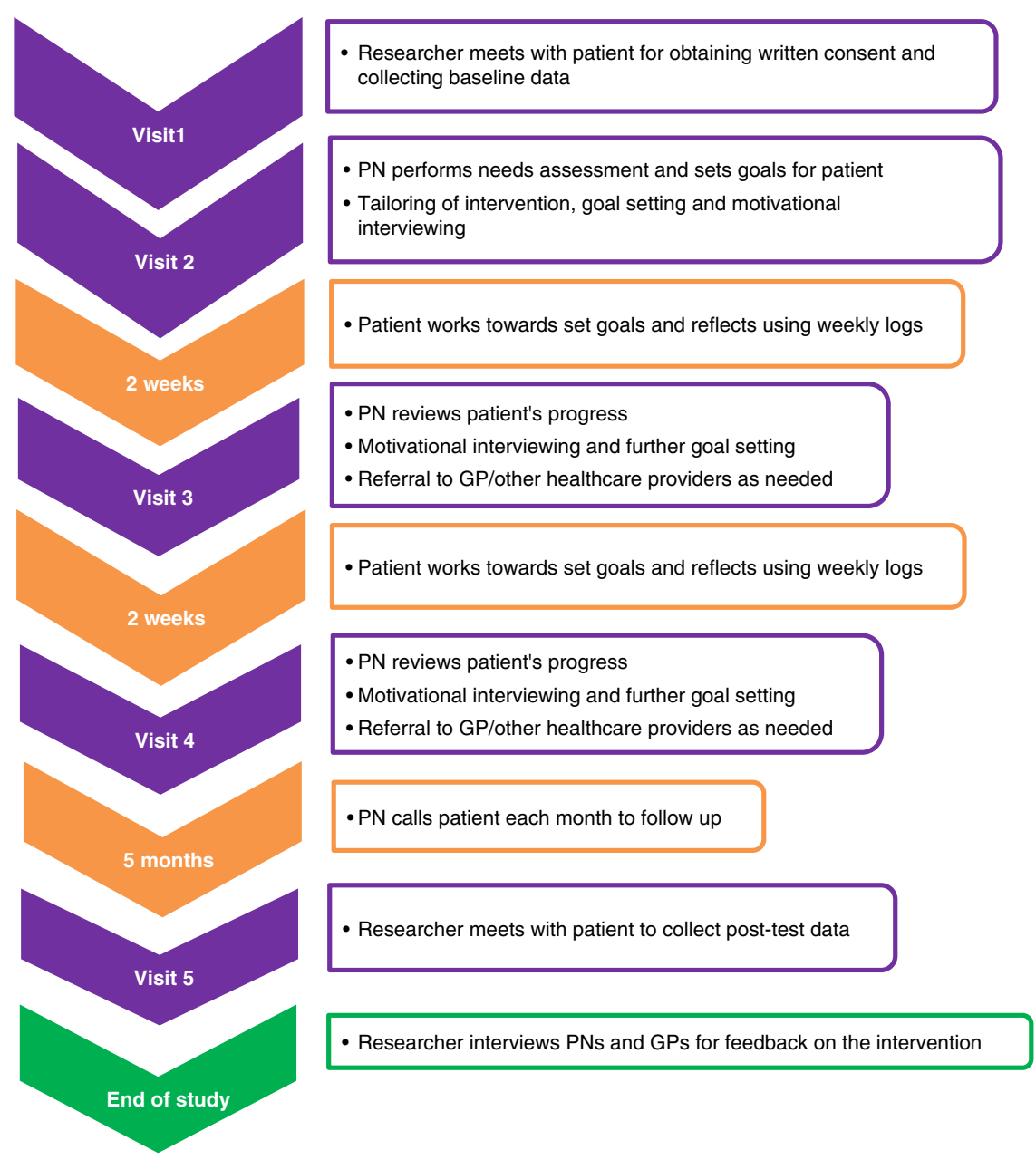

Fig. 1 Study protocol

Staff from participating practices will identify potential patients from their database based on inclusion criteria. PNs will then send an invitation letter to eligible patients and remind non-responders after two weeks by telephone. Interested patients will attend visit 1 at their practice, during which S.A. will answer questions, obtain written consent, and ask them to complete the baseline questionnaires.

Patient recruitment is deemed feasible as chronic respiratory disease accounts for $12 \%$ of patient-GP encounters in Australia, COPD being the $11^{\text {th }}$ most common cause for general practice visits. $^{18}$ Recent COPD casefinding studies in Sydney ${ }^{19}, 20$ have found high disease incidence.

\section{PN training}

PNs will attend a one-day work shop, facilitated by the investigators, which will cover basic pathophysiology of COPD, concept of multi-morbidity, the study design and rationale, COPD self-management based on GOLD ${ }^{21}$ (Global strategy for the diagnosis, management and prevention of COPD) and COPD- $X^{22}$ (Australian-New Zealand COPD guidelines), medication adherence, pulmonary rehabilitation, smoking cessation and principles of motivational interviewing. The workshop aims to equip PNs with knowledge and confidence to understand the intervention, and enable them to tailor and deliver it to patients using an assessment and planning template developed by the researchers. PNs' competency in delivering the intervention will be assessed using case scenarios, with investigators enacting patients and providing feedback. PNs' understanding of the intervention and feedback on the workshop will be obtained by means of an evaluation form.

\section{Intervention}

The self-management program of this study is based on the Health Belief Model (HBM) ${ }^{23}$ a widely-tested health education theory explaining human decision-making and subsequent behaviour.

The assessment and planning template for the PN-patient sessions incorporates the HBM's constructs with strategies for selfmanagement of COPD and co-morbidities. The template includes cues to:

1. COPD knowledge $\mathrm{e}^{24,25}$

2. Relationship between COPD and patients' co-morbidities

3. Management and prioritisation of their multiple chronic health conditions

4. Managing flare-ups using a COPD Action Plan ${ }^{26}$

5. Recommended exercises ${ }^{27}$ and pulmonary rehabilitation

6. Proper use of inhalers ${ }^{28,29}$

7. Importance of flu and pneumonia vaccination ${ }^{30,31}$

8. Medication review and adherence

9. Advice on overall health and wellbeing

10. Effects of smoking and benefits of quitting, assistance with quitting and abstinence ${ }^{32}$

Links to relevant resources will be uploaded to a website for the participants' reference. 
Table 1. Relationship between objectives, outcomes, measures and hypotheses

\begin{tabular}{|c|c|c|c|c|}
\hline Objective & Hypothesis & Outcome & Measure & Visit \\
\hline & \multirow{5}{*}{$\begin{array}{l}\text { Tailored self-management education will } \\
\text { improve patients' activation and engagement } \\
\text { in their health behaviour, knowledge and self- } \\
\text { efficacy of COPD, and increase confidence and } \\
\text { skills to manage their multi-morbidity }\end{array}$} & $\begin{array}{l}\text { Change in disease } \\
\text { knowledge }\end{array}$ & $\begin{array}{l}\text { COPD } \\
\text { Knowledge } \\
\text { Questionnaire } \\
\text { (COPD-Q) }\end{array}$ & 1,5 \\
\hline & & $\begin{array}{l}\text { Change in disease related } \\
\text { quality of life }\end{array}$ & $\begin{array}{l}\text { COPD } \\
\text { Assessment Test } \\
\text { (CAT) }\end{array}$ & 1,5 \\
\hline & & $\begin{array}{l}\text { Change in perceived impact } \\
\text { of multi-morbidity }\end{array}$ & $\begin{array}{l}\text { Multimorbidity } \\
\text { Illness } \\
\text { Perceptions Scale } \\
\text { (MULTIPleS) }^{39}\end{array}$ & 1,5 \\
\hline & & $\begin{array}{l}\text { Modified adherence to } \\
\text { prescribed medical regimen }\end{array}$ & $\begin{array}{l}\text { Morisky } \\
\text { Medication } \\
\text { Adherence Scale } \\
\text { (MMAS-8) }^{40}\end{array}$ & 1,5 \\
\hline & & $\begin{array}{l}\text { Accuracy of technique while } \\
\text { using inhalers prescribed for } \\
\text { COPD }\end{array}$ & $\begin{array}{l}\text { Inhaler device } \\
\text { technique } \\
\text { checklist }^{41}\end{array}$ & $\begin{array}{l}2,3 \\
4,5\end{array}$ \\
\hline $\begin{array}{l}\text { To assess the feasibility and } \\
\text { sustainability of the PN-delivered self- } \\
\text { management education program }\end{array}$ & & $\begin{array}{l}\text { Barriers and facilitators to } \\
\text { delivery of the intervention, } \\
\text { feedback on the program }\end{array}$ & $\begin{array}{l}\text { Semi-structured } \\
\text { interviews with } \\
\text { GPs and PNs }\end{array}$ & $\begin{array}{l}\text { End } \\
\text { of } \\
\text { study }\end{array}$ \\
\hline
\end{tabular}

During the first PN-patient session (visit 2), individual patient needs will be assessed, and the intervention tailored accordingly. In the following two sessions (visits 3 and 4), PNs will use motivational interviewing ${ }^{33}$ to address barriers faced by patients in managing their COPD in the face of co-morbidities, and work towards optimising health behaviour. The patients will maintain a daily log about their health behaviour in between the education sessions. The logistics of intervention delivery is based on the $5 \mathrm{~A}^{\prime} \mathrm{s}$ approach, ${ }^{34}$ which is an organisational tool devised to help clinicians Ask, Advise, Assess, Assist and Arrange for their patients' health behaviour.

Cues to action will be provided to patients in the form of motivational fridge magnets and five follow-up phone calls at monthly intervals by their PNs after the last session. During the course of the intervention, GPs will be notified of situations requiring medical attention (e.g., when treatment needs to be intensified) by the PNs.

\section{Outcome measures}

Impact of the program will be determined by change in patients' level of self-efficacy between baseline (visit 1) and six months' follow-up (visit 5), using the Patient Activation Measure (PAM) $13^{35}$ a highly-reliable, unidimensional scale of knowledge, skills and confidence for self-management of their health. ${ }^{36}$ The PAM classifies patients across four levels of activation, a significant difference being an increase or decrease of at least one level.

Secondary outcome measures include COPD Knowledge Questionnaire (COPD-Q), ${ }^{37}$ COPD Assessment Test (CAT, an indicator of COPD-related quality of life), ${ }^{38}$ Multimorbidity Illness Perceptions Scale (MULTIPleS, to detect change in perceived impact of multimorbidity), ${ }^{39}$ a COPD-specific version of the MULTIPleS, Morisky
Medication Adherence Scale (MMAS- 8$)^{40}$ and accuracy of inhaler technique. ${ }^{41}$ Relationship between objectives, hypothesis, outcomes and measures is outlined in Table 1.

In accordance with Normalisation Process Theory, ${ }^{42}$ a reliable framework for complex interventions, qualitative feedback will be obtained at the end of the study from participating PNs and GPs to examine feasibility and sustainability of the program.

\section{Data analysis}

Quantitative data will be analysed using Statistical Package for the Social Sciences. Descriptive statistics of responses, expressed as absolute and relative frequencies, will be generated. Categorical data will be analysed by Chi-squared or Fisher's exact test. Student's $t$-test for paired samples will be applied for comparing differences in pre and post-intervention outcome measures. Associations will be given as odds ratios.

Qualitative interview transcripts will be coded using NVivo software and thematically analysed by an iterative process.

\section{Sample size}

In accordance with the recommended sample size for feasibility studies demonstrating intervention efficacy in a single group, ${ }^{43}$ our pilot study requires 40 patients, allowing for a $20 \%$ drop-out rate ${ }^{44}$ between baseline and six-month visits. This is sufficient to detect a difference in paired measures of patient activation, as demonstrated by a recent study ${ }^{45}$ which used the PAM 13 as an outcome measure, with $85 \%$ power at the $5 \%$ significance level. A significant difference would be a change between two adjacent stages of the PAM. 
Qualitative process evaluation

Following completion of the program, participating PNs and GPs will be interviewed to gain their feedback. They will be invited via email and written informed consent obtained for recording the interviews, which will be conducted face-to-face by S.A. The interviews will be semi-structured with open ended questions covering their experience and opinions regarding the study, as well as feasibility and sustainability of the program. The interview transcripts will be analysed thematically and coded using NVivo software.

\section{DISCUSSION}

To our knowledge, this study is the first aimed at improving selfefficacy of primary care COPD patients with co-morbidities. Primary care is the ideal setting for implementing selfmanagement interventions because early-stage COPD is typically diagnosed and managed in this setting. Our tailored, PN-delivered education program might not only benefit these patients, but could also upskill the PNs' role in chronic disease management.

According to the UK Medical Research Council framework for complex interventions, ${ }^{46}$ feasibility studies like ours are a vital step for overcoming problems with acceptability, compliance, intervention delivery, participant recruitment, retention and follow-up, and effect size in larger intervention studies. Our study would be enriched by qualitative feedback from patient participants, but this will extend the data collection process and is hence a limitation.

Experience of intervention delivery and uptake from our pilot study would help refine the intervention and shed light on its acceptability and feasibility in day-to-day general practice, providing an evidence base for upscaling the intervention to be tested in a randomised controlled trial. This capacity-building initiative would also promote collaboration and knowledge translation between the various stakeholders.

\section{ACKNOWLEDGEMENTS}

This study is funded by GlaxoSmithKline Australia Pty Ltd (Study number 204950). S. A. is in receipt of a PhD scholarship for the study from the National Health and Medical Research Council (GNT1093032).

\section{COMPETING INTERESTS}

The authors declare no competing financial interests.

\section{REFERENCES}

1. World Health Organization. The top 10 causes of death. Fact sheet $N^{\circ} 310$. Updated May 2014.

2. Toelle, B. G., Xuan, W., Bird, T. E., et al. Respiratory symptoms and illness in older Australians: the burden of obstructive lung disease (BOLD) study. Med. J. Aust. 198, 144-148 (2013).

3. Ramond-Roquin, A. \& Fortin, M. Towards increased visibility of multimorbidity research. J. Comorbidity 6, 42-45 (2016).

4. Britt, H. C., Harrison, C. M., Miller, G. C. \& Knox, S. A. Prevalence and patterns of multimorbidity in Australia. Med. J. Aust. 189, 72-77 (2008).

5. Stoilkova, A., Janssen, D. J. A. \& Wouters, E. F. M. Educational programmes in COPD management interventions: a systematic review. Respir. Med. 107, 1637-1650 (2013).

6. Simmons, L. A., Wolever, R. Q., Bechard, E. M. \& Snyderman, R. Patient engagement as a risk factor in personalized health care: a systematic review of the literature on chronic disease. Genome Med. 6, 16 (2014).

7. Zwerink, M., Brusse-Keizer, M., van der Valk, P. D. L. P. M., et al. Self management for patients with chronic obstructive pulmonary disease (intervention review). Cochrane Database Syst. Rev.Art. No.: CD002990 (2014).

8. Bucknall, C. E., Miller, G., Lloyd, S. M., et al. Glasgow supported self-management trial (GSuST) for patients with moderate to severe COPD: randomised controlled trial. BMJ. 344, e1060 (2012).

9. Bischoff, E. W. M. A., Akkermans, R., Bourbeau, J., et al. Comprehensive self management and routine monitoring in chronic obstructive pulmonary disease patients in general practice: randomised controlled trial. BMJ. 345, e7642 (2012).
10. Mitchell, K. E., Johnson-Warrington, V., Apps, L. D., et al. A self-management programme for COPD: a randomised controlled trial. Eur. Respir. J. 44, 1538-1547 (2014).

11. Smith, S. M., Bayliss, E. A., Mercer, S. W., et al. How to design and evaluate interventions to improve outcomes for patients with multimorbidity. J. Comorbidity 3, 10-17 (2013).

12. Smith, S. M., Soubhi, H., Fortin, M., et al. Managing patients with multi-morbidity: systematic review of interventions in primary care and community settings. BMJ. 345, e5205 (2012).

13. Ansari, S., Hosseinzadeh, H., Dennis, S. \& Zwar, N. Patients' perspectives on impact of COPD diagnosis in the face of multi-morbidity - a qualitative study. Prim. Care Resp. Med. 24, 14036 (2014).

14. Gallagher, R., Roach, K., Belshaw, J., et al. A pre-test post-test study of a brief educational intervention demonstrates improved knowledge of potential acute myocardial infarction symptoms and appropriate responses in cardiac rehabilitation patients. Aust. Crit. Care 26, 49-54 (2013).

15. Zimmerman, B. W., Brown, S. T. \& Bowman, J. M. A self-management program for chronic obstructive pulmonary disease: relationship to dyspnea and self-efficacy. Rehabil. Nurs. 21, 253-257 (1996).

16. Bourbeau, J., Nault, D. \& Dang-Tan, T. Self-management and behaviour modification in COPD. Patient Educ. Couns. 52, 271-277 (2004).

17. Kirby,S. E., Dennis, S. M., Bazeley, P. \& Harris, M. F. Activating patients with chronic disease for self-management: comparison of self-managing patients with those managing by frequent readmissions to hospital. Aust. J. Prim. Health. 19, 198-206 (2013). doi:10.1071/PY12030.

18. Britt, H., Miller, G. C., Henderson, J., et al. BEACH (Bettering the Evaluation and Care of Health) General Practice Activity in Australia 2013-14. (Sydney University Press, November 2014).

19. Bunker, J., Hermiz, O., Zwar, N. \& Dennis, S. M. Feasibility and efficacy of COPD case finding by practice nurses. Aust. Fam. Physician 38, 826-830 (2009).

20. Bunker, J. M., Reddel, H. K., Dennis, S. M., et al. A pragmatic cluster randomized controlled trial of early intervention for chronic obstructive pulmonary disease by practice nurse-general practitioner teams: Study protocol. Implement. Sci. 7, 83 (2012).

21. Decramer, M., Agusti, A. G., Bourbeau, J., et al. Global strategy for the diagnosis, management, and prevention of chronic obstructive pulmonary disease (Updated 2016).

22. Yang, I., Dabscheck, E., George, J., et al. The COPD-X Plan: Australian and New Zealand Guidelines for the management of Chronic Obstructive Pulmonary Disease 2016. Version 2.47.

23. Hayden, J. A. Introduction to Health Behavior Theory. 1st edn; Ch. 4; 31-44 (Jones and Bartlett Publishers, 2009).

24. Lung Foundation Australia. COPD-The basics. http://lungfoundation.com.au/ wp-content/uploads/2014/07/LFA-COPD-The-Basics_24pp_0316_web.pdf.

25. Lung Foundation Australia. Better Living with Chronic Obstructive Pulmonary Disease-A Patient Guide. http://lungfoundation.com.au/wp-content/uploads/ 2014/02/Better-Living-with-COPD.pdf.

26. Lung Foundation Australia. COPD Action Plan. http://lungfoundation.com.au/wpcontent/uploads/2014/02/LFA-COPD-Action-Plan-Traffic-Lights_0515_ART-EditableFinal-15-05-15.pdf.

27. Exercise Is Medicine Australia Factsheet: Chronic Obstructive Pulmonary Disease and exercise. http://exerciseismedicine.org.au/wp-content/uploads/2014/05/ 2014-COPD-BRIEF.pdf.

28. National Asthma Council Australia. Using your inhaler. http://www. nationalasthma.org.au/how-to-videos/using-your-inhaler.

29. Lung Foundation Australia. Inhaler device technique. http://lungfoundation.com. au/patient-support/copd/inhaler-technique-fact-sheets/.

30. Australian Government Department of Health. Immunise Australia Program: Influenza (Flu). http://www.immunise.health.gov.au/internet/immunise/ publishing.nsf/Content/immunise-influenza.

31. Australian Government Department of Health. Immunise Australia Program: Pneumococcal disease. http://www.immunise.health.gov.au/internet/immunise/ publishing.nsf/Content/immunise-pneumococcal.

32. Australian Government. Quit Now. http://www.quitnow.gov.au/.

33. Linmans, J. J., van Rossem, C., Knottnerus, J. A. \& Spigt, M. Exploring the process when developing a lifestyle intervention in primary care for type 2 diabetes: a longitudinal process evaluation. Public Health 129, 52-59 (2015).

34. Dosh, S. A., Holtrop, J. S., Torres, T., et al. Changing organizational constructs into functional tools: an assessment of the $5 \mathrm{~A}$ 's in primary care practices. Ann. Fam. Med. 3, S50-S52 (2005).

35. Hibbard, J. H., Mahoney, E. R., Stockard, J. \& Tusler, M. Development and testing of a short form of the patient activation measure. Health Serv. Res. 40, 1918-1930 (2005).

36. Chubak, J., Anderson, M. L., Saunders, K. W., et al. Predictors of 1-year change in patient activation in older adults with diabetes mellitus and heart disease. J. Am. Geriatr. Soc. 60, 1316-1321 (2012). 
37. Ray, S. M., Helmer, R. S., Stevens, A. B., et al. Clinical utility of the chronic obstructive pulmonary disease knowledge questionnaire. Fam. Med. 45, 197-200 (2013).

38. Papaioannou, M., Pitsiou, G., Manika, K., et al. COPD assessment test: a simple tool to evaluate disease severity and response to treatment. COPD. 11, 489-495 (2014)

39. Gibbons, C. J., Kenning, C., Coventry, P. A., et al. Development of a multimorbidity illness perceptions scale (MULTIPleS). PLOS ONE 8, e81852 (2013).

40. Morisky, D. E., Green, L. W. \& Levine, D. M. Concurrent and predictive validity of a self-reported measure of medication adherence. Med. Care 24, 67-74 (1986).

41. Armour, C. L., LeMay, K., Saini, B., et al. Using the community pharmacy to identify patients at risk of poor asthma control and factors which contribute to this poor control. J. Asthma 48, 914-922 (2011).

42. Murray, E., Treweek, S., Pope, C., et al. Normalisation process theory: a framework for developing, evaluating and implementing complex interventions. BMC Med. 8, 63 (2010).

43. Hertzog, M. A. Considerations in determining sample size for pilot studies. Res. Nurs. Health 31, 180-191 (2008).

44. Armijo-Olivo, S., Warren, S. \& Magee, D. Intention to treat analysis, compliance, drop-outs and how to deal with missing data in clinical research: a review. Phys. Ther. Rev. 14, 1 (2009).
45. Evangelista, L. S., Liao, S., Motie, M., et al. On-going palliative care enhances perceived control and patient activation and reduces symptom distress in patients with symptomatic heart failure: A pilot study. Eur. J. Cardiovasc. Nurs. 13, 116-123 (2014)

46. Craig, P., Dieppe, P., Macintyre, S., et al. Developing and Evaluating Complex Interventions: New Guidance. Medical Research Council, UK. http://www.mrc.ac. uk/documents/pdf/complex-interventions-guidance/.

This work is licensed under a Creative Commons Attribution 4.0 International License. The images or other third party material in this article are included in the article's Creative Commons license, unless indicated otherwise in the credit line; if the material is not included under the Creative Commons license, users will need to obtain permission from the license holder to reproduce the material. To view a copy of this license, visit http://creativecommons.org/licenses/by/ $4.0 /$

(c) The Author(s) 2017 